\title{
Efficacy and Safety of Tocilizumab for Coronavirus Disease 2019 (COVID-19) Patients: A Systematic Review and Meta-analysis
}

\author{
Authors \\ Timotius Ivan Hariyanto' ${ }^{1}$, Willie Hardyson ${ }^{1}$, Andree Kurniawan²
}

\author{
Affiliations \\ 1 Faculty of Medicine, Pelita Harapan University, Karawaci, \\ Tangerang, Indonesia \\ 2 Department of Internal Medicine, Faculty of Medicine, \\ Pelita Harapan University, Karawaci, Tangerang, \\ Indonesia
}

Key words

coronavirus disease 2019, COVID-19, tocilizumab, immunomodulator, treatment

received 25.11.2020

accepted $\quad 07.12 .2020$

published online 05.01.2021

Bibliography

Drug Res 2021; 71: 265-274

DOI 10.1055/a-1336-2371

ISSN 2194-9379

(C) 2021. Thieme. All rights reserved.

Georg Thieme Verlag KG, Rüdigerstraße 14,

70469 Stuttgart, Germany

\section{Correspondence}

Andree Kurniawan

Department of Internal Medicine

Faculty of Medicine

Pelita Harapan University

Boulevard Jendral Sudirman Street

Karawaci

15811 Tangerang

Indonesia

Tel.: + 628158891655

andree.kurniawan@uph.edu

\section{ABSTRACT}

Background Currently, the data regarding the effectiveness and safety of tocilizumab as treatment for COVID-19 infection is still conflicting. This study aims to give clear evidence regarding the potential benefit and safety of tocilizumab in improving the outcome of COVID-19 patients.

Methods We systematically searched the PubMed and Europe PMC database using specific keywords related to our aims until November $1^{\text {st }}, 2020$. All articles published on COVID-19 and tocilizumab were retrieved. Statistical analysis was done using Review Manager 5.4 software.

Results A total of 38 studies with a total of 13412 COVID-19 patients were included in our analysis. Our meta-analysis showed that tocilizumab treatment is associated with reduction of mortality rate from COVID-19 [OR 0.54 (95\% Cl 0.42$0.71), p<0.00001, R^{2}=79 \%$, random-effect modelling], but did not alter the severity of COVID-19 [OR 1.05 (95\% Cl 0.92-1.20), $p=0.47, I^{2}=84 \%$, random-effect modelling] and length of hospital stay [Mean Difference 1.77 days ( $95 \% \mathrm{Cl}-0.61-4.14$ days), $p=0.15, R=97 \%$, random-effect modelling]. Tocilizumab also does not associated with serious adverse events compared with standard of care treatment [OR 0.91 ( $95 \% \mathrm{Cl} 0.71-1.15), p=0.42$, $R=46 \%$, random-effect modelling].

Conclusion Our study does not support the routine use of tocilizumab for COVID-19 patients. Future studies should focus more on other potential therapies for COVID-19 patients.

\section{Introduction}

Until now, the number of positive and death cases from coronavirus disease 2019 (COVID-19) is still increasing. This disease has caused significant health and economic burden across the world. The manifestations of the disease may vary from mild respiratory symptoms such as fever, nasal obstruction, and cough to severe life-threatening symptoms such as respiratory distress, shock, arrhythmia, and heart failure [1]. Several comorbid diseases has been demonstrated to be associated with severe COVID-19 infections, such as hypertension, diabetes, dyslipidemia, thyroid disease, cardiovascular disease, anemia, and pulmonary disease [2-4]. Currently, there are no widely accepted drugs for the management of COVID-19 patients. Several potential agents have been proposed to help in achieving faster recovery time and reducing the mortality rate in COVID-19 patients, and one of the agents is tocilizumab, an IL-6 inhibitor. Tocilizumab has been approved for the treatment of rheumatoid arthritis, juvenile idiopathic arthritis, and giant cell arteritis [5]. Recently, tocilizumab has been offered to help in re- 
ducing the pro-inflammatory cytokines in COVID-19 and preventing the cytokine storm syndrome that could contribute to the development of the severe outcome. Unfortunately, the evidence regarding the potential benefit and safety of tocilizumab in COVID-19 patients is still conflicting. Therefore, a meta-analysis is required to aid in solving this problem. This article aims to explore the efficacy and safety of tocilizumab administration in patients with COVID-19.

\section{Materials and Methods}

\section{Eligibility criteria}

Studies were included in this review if met the following inclusion criteria: representation for clinical questions (P: positive/confirmed cases of COVID-19; I: receiving tocilizumab as their treatment; C: did not receive tocilizumab or receive only standard of care treatment; O: efficacy of tocilizumab (rate of severe COVID-19, mortality, and length of hospital stay) and serious adverse events of tocilizumab (thromboembolism incident and secondary infection); S: type of study was a randomized control trial, cohort, clinical trial, case-cohort, and cross-over design) and if the full-text article was available. The following types of articles were excluded: articles other than original research (e. g., review articles or commentaries); case reports; articles not in the English language; articles on research in pediatric populations ( 17 years of age or younger); and articles on research in pregnant women.

\section{Search strategy and study selection}

A systematic search of the literature was conducted on PubMed and Europe PMC using the keywords "tocilizumab" OR "anti-IL-6" OR "IL-6 inhibitor" AND "coronavirus disease 2019” OR “COVID19", between 2019 and present time (November 1 1, 2020 ) with language restricted to English only. Duplicate results were removed. The remaining articles were independently screened for relevance by its abstracts with two authors. The full text of residual articles was assessed according to the inclusion and exclusion criteria. The references of all identified studies were also analyzed (forward and backward citation tracking) to identify other potentially eligible articles. The study was carried out per the Preferred Reporting Items for Systematic Reviews and Meta-Analyses (PRISMA) guidelines [6].

\section{Data extraction and quality assessment}

Data extraction was performed independently by two authors, we used standardized forms that include author, year, study design, number of participants, age, gender, number of patients who receive tocilizumab and who did not, tocilizumab dose, and proportion of patients with each outcome of COVID-19.

The outcome of interest was severe COVID-19, mortality, length of hospital stay, and serious adverse events which comprised of thromboembolism incident and secondary infection. Severe COVID-19 was defined as patients who had any of the following features at the time of, or after, admission: (1) respiratory distress ( $\geq 30$ breaths per $\mathrm{min}$ ); (2) oxygen saturation at rest $\leq 93 \%$; (3) ratio of the partial pressure of arterial oxygen ( $\mathrm{PaO} 2)$ to a fractional concentration of oxygen inspired air ( $\left.\mathrm{fiO}^{2}\right) \leq 300 \mathrm{mmHg}$; or (4) critical complication (respiratory failure, septic shock, and or multiple organ dysfunction/failure) or admission into ICU. Mortality outcome from COVID-19 was defined as the number of patients who were dead because of COVID-19 infection.

Two investigators independently evaluated the quality of the included cohort and case-control studies using the Newcastle-Ottawa Scale (NOS) [7]. The selection, comparability, and exposure of each study were broadly assessed and studies were assigned a score from zero to nine. Studies with scores $\geq 7$ were considered of good quality. They also independently evaluated the quality of the included clinical trial studies using the Revised Cochrane risk-ofbias tool for randomized trials (RoB 2) [8].

\section{Statistical analysis}

A meta-analysis was performed using Review Manager 5.4 (Cochrane Collaboration) software. Dichotomous variables were calculated using the Mantel-Haenszel formula with a random-effects model regardless of heterogeneity. The effect estimate was reported as risk ratio (RR) along with its $95 \%$ confidence intervals (Cls) for dichotomous variables, respectively. For continuous variables, the inverse variance method was used to obtain mean differences (MDs) and its standard deviations (SDs). P-value was twotailed, and the statistical significance was set at $\leq 0.05$. A funnel plot, Begg's rank correlation method [9], and Egger's weighted regression method [10] were adopted to statistically assess publication bias ( $P<0.05$ was considered statistically significant). When data were reported as medians and interquartile ranges, we would convert them to means and standard deviations for meta-analytical pooling using the formula by Wan X, et al [11].

\section{Results}

\section{Study selection and characteristics}

A total of 4274 records were obtained through systematic electronic searches and other ways. After the removal of duplicates, 4050 records remained. A total of 3956 records were excluded after screening the titles/abstracts because they did not match our inclusion and exclusion criteria. After evaluating 94 full-texts for eligibility, 54 full-text articles were excluded because they do not have the control/comparison group, 2 full-text articles were excluded because the articles were not in English, and finally, 38 studies [12-49] with a total of 13412 COVID-19 patients were included in the meta-analysis ( $\triangleright$ Fig. 1). Of a total of 38 included studies, 3 were double-blind randomized-controlled trial (RCT), 4 were openlabel RCT, 23 were retrospective cohort, 3 studies were prospective cohort, while the remaining 5 studies was a case-control study. The dose and preparation of tocilizumab used were varied among the included studies. Most of the included studies ( 24 studies) use intravenous tocilizumab at dosage $8 \mathrm{mg} / \mathrm{kg}, 1-2$ doses, while the remaining studies use tocilizumab at $400 \mathrm{mg}, 1-2$ doses, and subcutaneous tocilizumab at a dosage of $324 \mathrm{mg}$ given as two consecutive injections. The essential characteristics of the included studies are summarized in $>$ Table $\mathbf{1}$. 


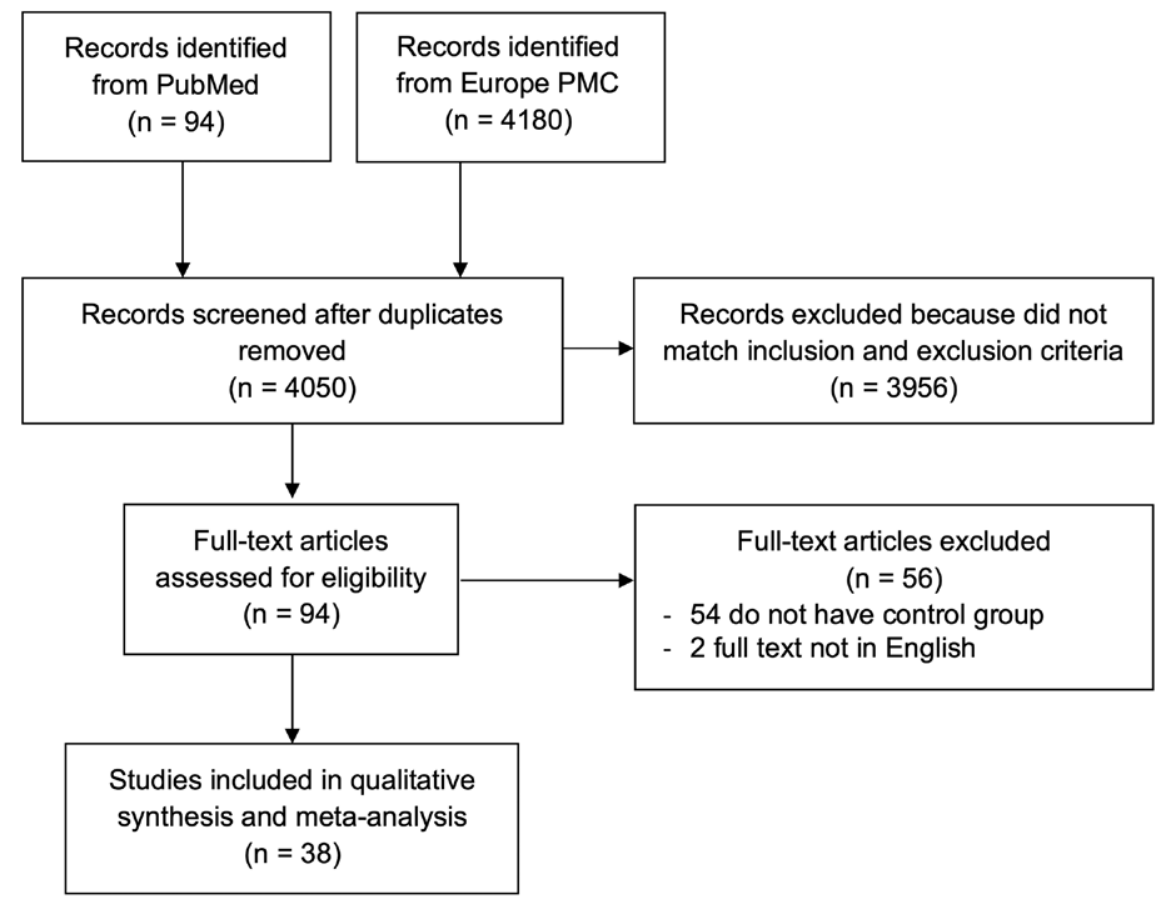

- Fig. 1 PRISMA diagram of the detailed process of selection of studies for inclusion in the systematic review and meta-analysis.

- Table 1 Characteristics of included studies.

\begin{tabular}{|c|c|c|c|c|c|c|c|}
\hline \multirow[t]{2}{*}{ Study } & \multirow{2}{*}{$\begin{array}{l}\text { Sample } \\
\text { size }\end{array}$} & \multirow[t]{2}{*}{ Design } & \multirow[t]{2}{*}{ Tocilizumab dose } & \multicolumn{2}{|c|}{ Tocilizumab patients } & \multicolumn{2}{|c|}{ Non-tocilizumab patients } \\
\hline & & & & n (\%) & Age (years) & n (\%) & $\begin{array}{l}\text { Age } \\
\text { (years) }\end{array}$ \\
\hline $\begin{array}{l}\text { Campochiaro C } \\
\text { et al. [12] } 2020\end{array}$ & 65 & $\begin{array}{l}\text { Retrospective } \\
\text { cohort }\end{array}$ & IV: $400 \mathrm{mg}, 1-2$ doses & $32(49.2 \%)$ & $64 \pm 16.2$ & $33(50.8 \%)$ & $63.5 \pm 15.1$ \\
\hline $\begin{array}{l}\text { Canziani LM } \\
\text { et al. [13] } 2020\end{array}$ & 128 & Case-control & IV: $8 \mathrm{mg} / \mathrm{kg}, 1-2$ doses & $64(50 \%)$ & $63 \pm 12$ & $64(50 \%)$ & $64 \pm 8$ \\
\hline $\begin{array}{l}\text { Capra R } \\
\text { et al. [14] } 2020\end{array}$ & 85 & $\begin{array}{l}\text { Retrospective } \\
\text { cohort }\end{array}$ & IV: $400 \mathrm{mg}, 1$ dose & $62(72.9 \%)$ & $63.3 \pm 14.1$ & $23(27.1 \%)$ & $68.3 \pm 18.5$ \\
\hline $\begin{array}{l}\text { Chilimuri S } \\
\text { et al. [15] } 2020\end{array}$ & 1225 & $\begin{array}{l}\text { Retrospective } \\
\text { cohort }\end{array}$ & IV: $400 \mathrm{mg}, 1-2$ doses & $87(7.1 \%)$ & $61.6 \pm 15.5$ & $1138(92.9 \%)$ & $63 \pm 14.8$ \\
\hline $\begin{array}{l}\text { Colaneri M } \\
\text { et al. [16] } 2020\end{array}$ & 112 & $\begin{array}{l}\text { Retrospective } \\
\text { cohort }\end{array}$ & IV: $400 \mathrm{mg}, 1$ dose & $21(18.7 \%)$ & $62.3 \pm 18.6$ & $91(81.3 \%)$ & $63.7 \pm 16.3$ \\
\hline $\begin{array}{l}\text { De Rossi } \\
\text { et al. [17] } 2020\end{array}$ & 158 & $\begin{array}{l}\text { Retrospective } \\
\text { cohort }\end{array}$ & $\begin{array}{l}\text { IV: } 400 \mathrm{mg}, 1 \text { dose } \\
\text { SC: } 324 \mathrm{mg}, 1 \text { dose }\end{array}$ & $90(56.9 \%)$ & $62.9 \pm 12.5$ & $68(43.1 \%)$ & $71 \pm 14.6$ \\
\hline $\begin{array}{l}\text { Eimer J } \\
\text { et al. [18] } 2020\end{array}$ & 87 & $\begin{array}{l}\text { Retrospective } \\
\text { cohort }\end{array}$ & IV: $8 \mathrm{mg} / \mathrm{kg}, 1-2$ doses & $29(33.3 \%)$ & $56.6 \pm 10.3$ & $58(66.7 \%)$ & $57.2 \pm 9.4$ \\
\hline $\begin{array}{l}\text { Enzmann MO } \\
\text { et al. [19] } 2020\end{array}$ & 150 & $\begin{array}{l}\text { Retrospective } \\
\text { cohort }\end{array}$ & IV: $8 \mathrm{mg} / \mathrm{kg}, 1-2$ doses & $12(15.3 \%)$ & $\mathrm{N} / \mathrm{A}$ & $66(84.7 \%)$ & $\mathrm{N} / \mathrm{A}$ \\
\hline $\begin{array}{l}\text { Gokhale Y } \\
\text { et al. [20] } 2020\end{array}$ & 269 & $\begin{array}{l}\text { Retrospective } \\
\text { cohort }\end{array}$ & IV: $400 \mathrm{mg}, 1$ dose & $151(56.1 \%)$ & $52.3 \pm 11.8$ & $118(43.9 \%)$ & $55.3 \pm 12.5$ \\
\hline $\begin{array}{l}\text { Guaraldi G } \\
\text { et al. [21] } 2020\end{array}$ & 544 & $\begin{array}{l}\text { Retrospective } \\
\text { cohort }\end{array}$ & $\begin{array}{l}\text { IV: } 8 \mathrm{mg} / \mathrm{kg}, 2 \text { doses } \\
\text { SC: } 162 \mathrm{mg}, 2 \text { doses }\end{array}$ & $179(32.9 \%)$ & $63.3 \pm 13.3$ & $365(67.1 \%)$ & $68 \pm 15.5$ \\
\hline $\begin{array}{l}\text { Gupta S } \\
\text { et al. [22] } 2020\end{array}$ & 3924 & $\begin{array}{l}\text { Retrospective } \\
\text { cohort }\end{array}$ & IV: $8 \mathrm{mg} / \mathrm{kg}, 1-2$ doses & $433(11 \%)$ & $57 \pm 12.5$ & $3491(89 \%)$ & $62.3 \pm 14.8$ \\
\hline $\begin{array}{l}\text { Hermine O } \\
\text { et al. [23] } 2020\end{array}$ & 130 & Open-label RCT & IV: $8 \mathrm{mg} / \mathrm{kg}, 1-2$ doses & $63(48.6 \%)$ & $65.1 \pm 12.7$ & $67(51.4 \%)$ & $64.2 \pm 11.2$ \\
\hline $\begin{array}{l}\text { Holt GE } \\
\text { et al. [24] } 2020\end{array}$ & 62 & $\begin{array}{l}\text { Retrospective } \\
\text { cohort }\end{array}$ & IV: $400 \mathrm{mg}, 1$ dose & $32(51.6 \%)$ & $\mathrm{N} / \mathrm{A}$ & $30(48.4 \%)$ & $\mathrm{N} / \mathrm{A}$ \\
\hline
\end{tabular}


- Table 1 Continued.

\begin{tabular}{|c|c|c|c|c|c|c|c|}
\hline \multirow[t]{2}{*}{ Study } & \multirow{2}{*}{$\begin{array}{l}\text { Sample } \\
\text { size }\end{array}$} & \multirow[t]{2}{*}{ Design } & \multirow[t]{2}{*}{ Tocilizumab dose } & \multicolumn{2}{|c|}{ Tocilizumab patients } & \multicolumn{2}{|c|}{ Non-tocilizumab patients } \\
\hline & & & & n (\%) & Age (years) & n (\%) & $\begin{array}{l}\text { Age } \\
\text { (years) }\end{array}$ \\
\hline $\begin{array}{l}\text { Ip A } \\
\text { et al. [25] } 2020\end{array}$ & 547 & $\begin{array}{l}\text { Retrospective } \\
\text { cohort }\end{array}$ & IV: $400 \mathrm{mg}, 1$ dose & $134(24.4 \%)$ & $61.6 \pm 12.5$ & $413(75.6 \%)$ & $68 \pm 14.1$ \\
\hline $\begin{array}{l}\text { Kewan T } \\
\text { et al. [26] } 2020\end{array}$ & 51 & $\begin{array}{l}\text { Retrospective } \\
\text { cohort }\end{array}$ & $\mathrm{IV}: 8 \mathrm{mg} / \mathrm{kg}, 1$ dose & $28(54.9 \%)$ & $62 \pm 13.3$ & $23(45.1 \%)$ & $66.6 \pm 14.8$ \\
\hline $\begin{array}{l}\text { Klopfenstein T } \\
\text { et al. [27] } 2020\end{array}$ & 206 & Case-control & IV: $8 \mathrm{mg} / \mathrm{kg}, 1-2$ doses & $30(14.5 \%)$ & $75.6 \pm 11.3$ & $176(85.5 \%)$ & $74.3 \pm 11$ \\
\hline $\begin{array}{l}\text { Lengnan X } \\
\text { et al. [28] } 2020\end{array}$ & 19 & $\begin{array}{l}\text { Retrospective } \\
\text { cohort }\end{array}$ & $\mathrm{IV}: 400 \mathrm{mg}, 1$ dose & $5(26.3 \%)$ & $73.2 \pm 4.4$ & $14(73.7 \%)$ & $66.2 \pm 5$ \\
\hline $\begin{array}{l}\text { Masia M } \\
\text { et al. [29] } 2020\end{array}$ & 138 & $\begin{array}{l}\text { Prospective } \\
\text { cohort }\end{array}$ & $\begin{array}{l}\text { IV: } 400 \mathrm{mg} \text { if }<75 \mathrm{~kg} \text { and } \\
600 \mathrm{mg} \text { if } \geq 75 \mathrm{~kg}\end{array}$ & $76(55 \%)$ & $65.2 \pm 14.9$ & $62(45 \%)$ & $65.9 \pm 16.8$ \\
\hline $\begin{array}{l}\text { Martinez-Sanz ] } \\
\text { et al. [30] } 2020\end{array}$ & 1229 & $\begin{array}{l}\text { Retrospective } \\
\text { cohort }\end{array}$ & IV: $8 \mathrm{mg} / \mathrm{kg}, 1-2$ doses & $260(21.1 \%)$ & $65.3 \pm 15.5$ & $969(78.9 \%)$ & $68.3 \pm 17$ \\
\hline $\begin{array}{l}\text { Menzella F } \\
\text { et al. [31] } 2020\end{array}$ & 79 & $\begin{array}{l}\text { Prospective } \\
\text { cohort }\end{array}$ & $\begin{array}{l}\text { IV: } 8 \mathrm{mg} / \mathrm{kg}, 2 \text { doses } \\
\text { SC: } 162 \mathrm{mg}, 2-4 \text { doses }\end{array}$ & $41(51.8 \%)$ & $63.3 \pm 10.6$ & $38(48.2 \%)$ & $70.3 \pm 11.3$ \\
\hline $\begin{array}{l}\text { Mikulska M } \\
\text { et al. [32] } 2020\end{array}$ & 196 & $\begin{array}{l}\text { Prospective } \\
\text { cohort }\end{array}$ & $\begin{array}{l}\text { IV: } 8 \mathrm{mg} / \mathrm{kg}, 1-2 \text { doses } \\
\text { SC: } 162 \mathrm{mg}, 1-2 \text { doses }\end{array}$ & $130(66.3 \%)$ & $64.5 \pm 12.4$ & $66(33.7 \%)$ & $73.5 \pm 14.4$ \\
\hline $\begin{array}{l}\text { Moiseev S } \\
\text { et al. [33] } 2020\end{array}$ & 137 & $\begin{array}{l}\text { Retrospective } \\
\text { cohort }\end{array}$ & IV: $400 \mathrm{mg}, 1$ dose & $83(60.5 \%)$ & $55.6 \pm 11.1$ & $54(39.5 \%)$ & $56.3 \pm 14$ \\
\hline $\begin{array}{l}\text { Moreno-Perez O } \\
\text { et al. [34] } 2020\end{array}$ & 236 & $\begin{array}{l}\text { Retrospective } \\
\text { cohort }\end{array}$ & IV: $8 \mathrm{mg} / \mathrm{kg}, 1-2$ doses & 77 (32.6\%) & $62.3 \pm 14$ & $159(67.4 \%)$ & $57 \pm 19.2$ \\
\hline $\begin{array}{l}\text { Perrone F } \\
\text { et al. [35] } 2020\end{array}$ & 301 & Open-label RCT & IV: $8 \mathrm{mg} / \mathrm{kg}, 1-2$ doses & $180(59.8 \%)$ & $\mathrm{N} / \mathrm{A}$ & $121(40.2 \%)$ & $\mathrm{N} / \mathrm{A}$ \\
\hline $\begin{array}{l}\text { Potere N } \\
\text { et al. [36] } 2020\end{array}$ & 80 & Case-control & SC: $162 \mathrm{mg}, 2$ doses & $40(50 \%)$ & $59.8 \pm 16.9$ & $40(50 \%)$ & $59.1 \pm 17$ \\
\hline $\begin{array}{l}\text { Price CC } \\
\text { et al. [37] } 2020\end{array}$ & 239 & $\begin{array}{l}\text { Retrospective } \\
\text { cohort }\end{array}$ & IV: $8 \mathrm{mg} / \mathrm{kg}, 1-2$ doses & $153(64 \%)$ & $\mathrm{N} / \mathrm{A}$ & $86(46 \%)$ & $\mathrm{N} / \mathrm{A}$ \\
\hline $\begin{array}{l}\text { Rodriguez-Bano ] } \\
\text { et al. [38] } 2020\end{array}$ & 432 & $\begin{array}{l}\text { Retrospective } \\
\text { cohort }\end{array}$ & IV: $8 \mathrm{mg} / \mathrm{kg}, 1-2$ doses & $88(20.3 \%)$ & $64.6 \pm 11.8$ & $344(79.7 \%)$ & $68 \pm 12.5$ \\
\hline $\begin{array}{l}\text { Rojas-Marte G } \\
\text { et al. [39] } 2020\end{array}$ & 193 & Case-control & IV: $8 \mathrm{mg} / \mathrm{kg}, 1-2$ doses & $96(49.7 \%)$ & $58.8 \pm 13.6$ & $97(50.3 \%)$ & $62 \pm 14$ \\
\hline $\begin{array}{l}\text { Roomi S } \\
\text { et al. [40] } 2020\end{array}$ & 176 & $\begin{array}{l}\text { Retrospective } \\
\text { cohort }\end{array}$ & IV: $8 \mathrm{mg} / \mathrm{kg}, 1-2$ doses & $134(78.8 \%)$ & $65.4 \pm 10.5$ & $36(21.2 \%)$ & $58 \pm 13.2$ \\
\hline $\begin{array}{l}\text { Rosas I } \\
\text { et al. [41] } 2020\end{array}$ & 438 & $\begin{array}{l}\text { Double-blind } \\
\text { RCT }\end{array}$ & IV: $8 \mathrm{mg} / \mathrm{kg}, 1-2$ doses & $294(67.1 \%)$ & $60.9 \pm 14.6$ & $144(32.9 \%)$ & $60.6 \pm 13.7$ \\
\hline $\begin{array}{l}\text { Rossi B } \\
\text { et al. [42] } 2020\end{array}$ & 246 & Case-control & IV: $400 \mathrm{mg}, 1$ dose & $106(43 \%)$ & $64.3 \pm 13$ & $140(57 \%)$ & $70.1 \pm 16.5$ \\
\hline $\begin{array}{l}\text { Roumier M } \\
\text { et al. [43] } 2020\end{array}$ & 59 & $\begin{array}{l}\text { Retrospective } \\
\text { cohort }\end{array}$ & IV: $8 \mathrm{mg} / \mathrm{kg}, 1-2$ doses & $30(50.8 \%)$ & $58.8 \pm 12.4$ & $29(49.2 \%)$ & $71.2 \pm 15.4$ \\
\hline $\begin{array}{l}\text { Ruiz-Antoran B } \\
\text { et al. [44] } 2020\end{array}$ & 506 & $\begin{array}{l}\text { Retrospective } \\
\text { cohort }\end{array}$ & IV: $8 \mathrm{mg} / \mathrm{kg}, 1-2$ doses & $268(52.9 \%)$ & $65 \pm 11.7$ & $238(47.1 \%)$ & $71.3 \pm 14.2$ \\
\hline $\begin{array}{l}\text { Salama C } \\
\text { et al. [45] } 2020\end{array}$ & 377 & $\begin{array}{l}\text { Double-blind } \\
\text { RCT }\end{array}$ & IV: $8 \mathrm{mg} / \mathrm{kg}, 1-2$ doses & $249(66 \%)$ & $56 \pm 14.3$ & $128(34 \%)$ & $55.6 \pm 14.9$ \\
\hline $\begin{array}{l}\text { Salvarani C } \\
\text { et al. [46] } 2020\end{array}$ & 126 & Open-label RCT & IV: $8 \mathrm{mg} / \mathrm{kg}, 1-2$ doses & $60(47.6 \%)$ & $62.1 \pm 16.2$ & $66(52.4 \%)$ & $61.6 \pm 14$ \\
\hline $\begin{array}{l}\text { Somers EC } \\
\text { et al. [47] } 2020\end{array}$ & 154 & $\begin{array}{l}\text { Retrospective } \\
\text { cohort }\end{array}$ & $\mathrm{IV}: 8 \mathrm{mg} / \mathrm{kg}, 1$ dose & $78(50.6 \%)$ & $55 \pm 14.9$ & $76(49.4 \%)$ & $60 \pm 14.5$ \\
\hline $\begin{array}{l}\text { Stone JH } \\
\text { et al. [48] } 2020\end{array}$ & 243 & $\begin{array}{l}\text { Double-blind } \\
\text { RCT }\end{array}$ & IV: $8 \mathrm{mg} / \mathrm{kg}, 1$ dose & $161(66.2 \%)$ & $59.2 \pm 17.2$ & $82(33.8 \%)$ & $56.3 \pm 17.1$ \\
\hline $\begin{array}{l}\text { Wang D } \\
\text { et al. [49] } 2020\end{array}$ & 65 & Open-label RCT & IV: $400 \mathrm{mg}, 1-2$ doses & $34(52.3 \%)$ & $64.1 \pm 9.6$ & $31(47.7 \%)$ & $62 \pm 11.1$ \\
\hline
\end{tabular}

\section{Quality of study assessment}

Studies with various study designs including a clinical trial, cohort, and case-control were included in this review and assessed accord- ingly with the appropriate scale or tool. Newcastle Ottawa Scales (NOS) were used to assess the cohort and case-control studies ( $\vee$ Table 2). All included studies were rated 'good'. For clinical trial 
- Table 2 Newcastle-Ottawa quality assessment of observational studies.

\begin{tabular}{|c|c|c|c|c|c|c|}
\hline First author, year & Study design & Selection & $\begin{array}{l}\text { Comparabil- } \\
\text { ity }\end{array}$ & Outcome & Total score & Result \\
\hline Campochiaro C et al. [12] 2020 & Cohort & $* * * *$ & ${ }^{* *}$ & ${ }^{* * *}$ & 9 & Good \\
\hline Canziani LM et al. [13] 2020 & Cohort & $* * * *$ & $* *$ & $* * *$ & 9 & Good \\
\hline Capra R et al. [14] 2020 & Cohort & $* * *$ & $* *$ & $* *$ & 7 & Good \\
\hline Chilimuri S et al. [15] 2020 & Cohort & $* * *$ & $* *$ & $* * *$ & 8 & Good \\
\hline Colaneri M et al. [16] 2020 & Cohort & $* * * *$ & $* *$ & $* * *$ & 9 & Good \\
\hline De Rossi N et al. [17] 2020 & Cohort & $* * * *$ & $* *$ & $* * *$ & 9 & Good \\
\hline Eimer J et al. [18] 2020 & Cohort & $* *$ & $* *$ & $* * *$ & 7 & Good \\
\hline Enzmann MO et al. [19] 2020 & Cohort & $* *$ & $* *$ & $* * *$ & 7 & Good \\
\hline Gokhale Y et al. [20] 2020 & Cohort & $* * *$ & $* *$ & $* * *$ & 8 & Good \\
\hline Guaraldi et al. [21] 2020 & Cohort & $* * * *$ & $* *$ & $* * *$ & 9 & Good \\
\hline Gupta S et al. [22] 2020 & Cohort & $* * * *$ & $* *$ & $* * *$ & 9 & Good \\
\hline Holt GE et al. [24] 2020 & Cohort & $* *$ & $* *$ & $* * *$ & 7 & Good \\
\hline Ip A et al. [25] 2020 & Cohort & ${ }^{* * *}$ & $* *$ & $* * *$ & 8 & Good \\
\hline Kewan T et al. [26] 2020 & Cohort & $* * *$ & $* *$ & $* * *$ & 8 & Good \\
\hline Klopfenstein T et al. [27] 2020 & Cohort & $* * *$ & $* *$ & $* *$ & 7 & Good \\
\hline Lengnan X et al. [28] 2020 & Cohort & $* * *$ & $* *$ & $* * *$ & 8 & Good \\
\hline Masia M et al. [29] 2020 & Cohort & $* * *$ & $* *$ & $* * *$ & 8 & Good \\
\hline Martinez-Sanz ] et al. [30] 2020 & Cohort & $* * * *$ & $* *$ & $* * *$ & 9 & Good \\
\hline Menzella F et al. [31] 2020 & Cohort & ${ }^{* * *}$ & $* *$ & $* * *$ & 8 & Good \\
\hline Mikulska M et al. [32] 2020 & Cohort & $* * * *$ & $* *$ & $* * *$ & 9 & Good \\
\hline Moiseev S et al. [33] 2020 & Cohort & $* *$ & $* *$ & $* * *$ & 7 & Good \\
\hline $\begin{array}{l}\text { Moreno-Perez O et al. [34] } \\
2020\end{array}$ & Cohort & $* *$ & $* *$ & *** & 7 & Good \\
\hline Potere N et al. [36] 2020 & Cohort & $* * *$ & $* *$ & $* *$ & 7 & Good \\
\hline Price CC et al. [37] 2020 & Cohort & $* * *$ & $* *$ & $* * *$ & 8 & Good \\
\hline $\begin{array}{l}\text { Rodriguez-Bano J et al. [38] } \\
2020\end{array}$ & Cohort & $* * * *$ & $* *$ & $* * *$ & 9 & Good \\
\hline Rojas-Marte G et al. [39] 2020 & Case-control & $* * *$ & $* *$ & $* * *$ & 8 & Good \\
\hline Roomi S et al. [40] 2020 & Cohort & $* * *$ & $* *$ & $* * *$ & 8 & Good \\
\hline Rossi B et al. [42] 2020 & Case-control & $* * *$ & $* *$ & $* * *$ & 8 & Good \\
\hline Roumier M et al. [43] 2020 & Cohort & $* * *$ & $* *$ & $* *$ & 7 & Good \\
\hline Ruiz-Antoran B et al. [44] 2020 & Cohort & $* * *$ & $* *$ & $* * *$ & 8 & Good \\
\hline Salama C et al. [45] 2020 & Cohort & $* * *$ & $* *$ & $* * *$ & 8 & Good \\
\hline Somers EC et al. [46] 2020 & Cohort & $* * *$ & $* *$ & $* * *$ & 8 & Good \\
\hline
\end{tabular}

studies, the Revised Cochrane risk-of-bias tool for randomized trials (RoB 2) was used and all of the included trials showed a low risk of bias ( $\triangleright$ Table 3 ). In conclusion, all studies were seemed fit to be included in the meta-analysis.

\section{Tocilizumab and outcomes}

\section{Tocilizumab efficacy}

Our pooled analysis showed that tocilizumab administration was associated with reduction of mortality rate from COVID-19 [OR 0.54 (95\% Cl 0.42-0.71), $p<0.00001,1^{2}=79 \%$, random-effect modelling] ( $\triangleright$ Fig. 2a). However, tocilizumab administration did not alter the severity of COVID-19 [OR 1.05 (95\% Cl 0.92-1.20), $p=0.47, P=84 \%$, random-effect modelling] ( $>$ Fig. 2 b) and length of hospital stay [Mean Difference 1.77 days (95\% Cl - 0.61-4.14 days), $p=0.15, R^{2}=97 \%$, random-effect modelling] ( $\vdash$ Fig. $2 \mathbf{c}$ ).

\section{Tocilizumab safety}

Our meta-analysis showed that tocilizumab administration was not associated with serious adverse events [OR 0.91 ( $95 \% \mathrm{Cl} 0.71-$ 1.15), $p=0.42, R=46 \%$, random-effect modelling] ( $>$ Fig. 2d). Subgroup analysis showed that tocilizumab administration was not associated with thromboembolism incident [OR 1.02 (95\% Cl 0.69$1.50), p=0.93, R=12 \%$, random-effect modelling], nor secondary infection [OR $0.86(95 \% \mathrm{Cl} 0.63-1.18), p=0.36, R=57 \%$, randomeffect modelling].

\section{Subgroup analysis}

Subgroup analysis for clinical trial studies showed a higher OR for mortality rate outcome [OR 0.90 (95\% Cl 0.64-1.26), $p=0.54$, $R=0 \%$, random-effect modelling] compared to observational studies [OR 0.50 (95\% Cl 0.38-0.67), $p<0.00001, R^{2}=80 \%$, random-ef- 
- Table 3 Risk of bias assessment for clinical trial studies using RoB-2 tool.

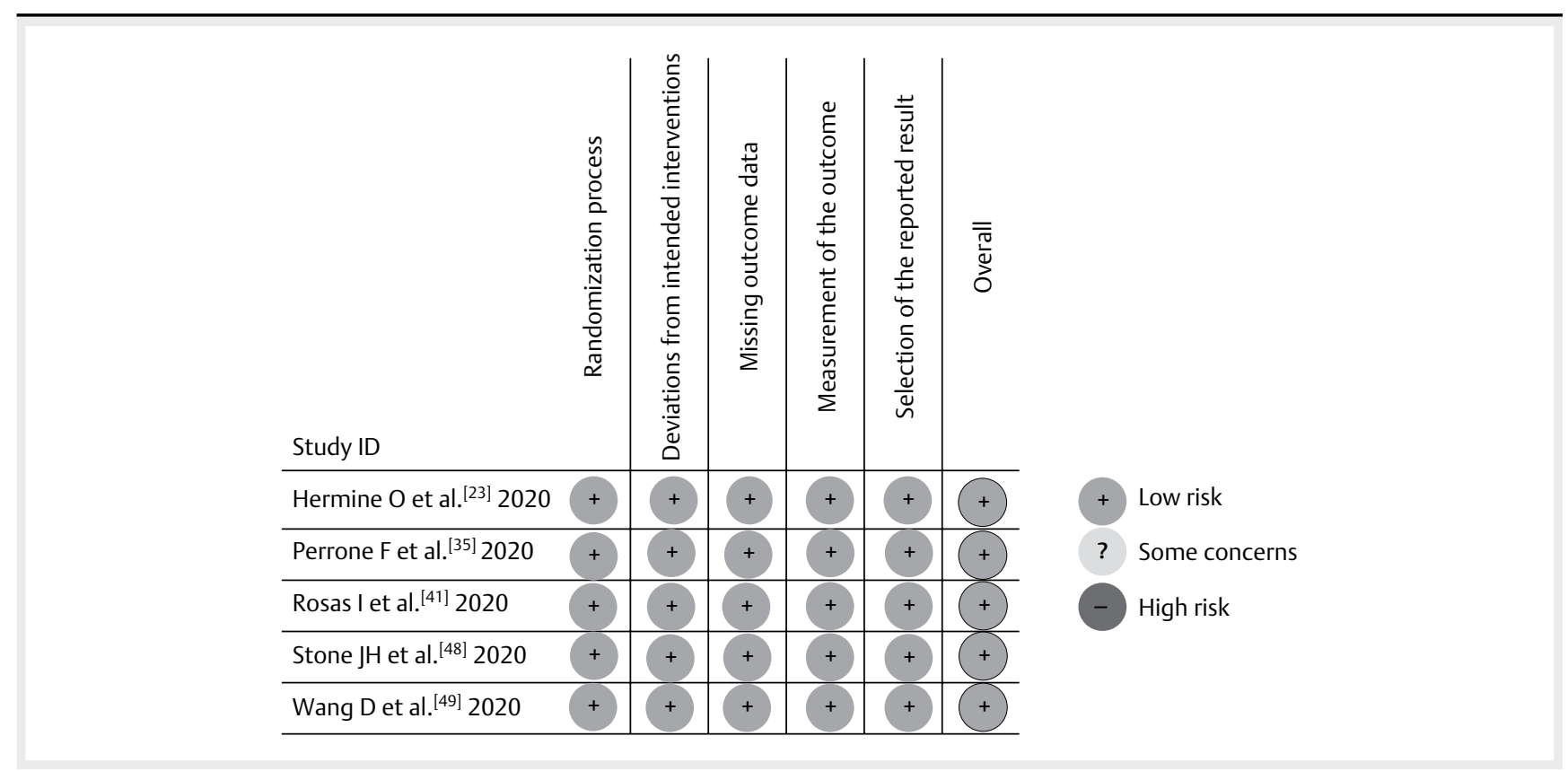

> Table 4 Summary of meta-analysis.

\begin{tabular}{|c|c|c|c|c|c|}
\hline Outcomes & $\begin{array}{l}\text { Effect size (95\% Confidence Interval), } \\
\text { p-value }\end{array}$ & $\begin{array}{l}\text { Heterogeneity }\left(I^{2}\right), \\
p \text {-value }\end{array}$ & Begg's test & Egger's test & $\begin{array}{l}\text { Number of } \\
\text { Studies }\end{array}$ \\
\hline Mortality & $\mathrm{OR}=0.54[0.42-0.71],<0.00001$ & $79 \%,<0.00001$ & 0.968 & 0.284 & 37 \\
\hline Severe COVID-19 & $\mathrm{OR}=1.05[0.92-1.20], 0.47$ & $84 \%,<0.00001$ & 0.464 & 0.150 & 30 \\
\hline Length of hospital stay & Mean Difference $=1.77[-0.61-4.14], 0.15$ & $97 \%,<0.00001$ & 0.836 & 0.213 & 17 \\
\hline Thrombosis incident & $\mathrm{OR}=1.02[0.69-1.50], 0.93$ & $12 \%, 0.33$ & 0.916 & 0.978 & 9 \\
\hline Secondary infection & $\mathrm{OR}=0.86[0.63-1.18], 0.36$ & $57 \%, 0.02$ & 0.558 & 0.451 & 16 \\
\hline
\end{tabular}

fect modelling]. Subgroup analysis for clinical trial studies showed a lower OR for severe COVID-19 outcome [OR 0.81 (95\% Cl 0.531.23), $p=0.32, I^{2}=23 \%$, random-effect modelling] compared to observational studies [OR 1.11 (95\% Cl 0.96-1.28), $p=0.15$, $R=86 \%$, random-effect modelling]. Subgroup analysis for clinical trial studies showed a lower Mean Difference for length of hospital stay outcome [Mean Difference -1.43 days (95\% Cl - 5.13-2.26 days), $p=0.45, R^{2}=95 \%$, random-effect modelling] compared to observational studies [Mean Difference 2.70 days $(95 \% \mathrm{Cl}-0.59-$ 5.99 days), $p=0.11, R^{2}=97 \%$, random-effect modelling]. Subgroup analysis for clinical trial studies showed a lower OR for serious adverse events outcome [OR 0.52 (95\% Cl 0.29-0.92), $p=0.02$, $R^{2}=38 \%$, random-effect modelling] compared to observational studies [OR 1.04 (95\% Cl 0.80-1.35), $p=0.76, P^{2}=41 \%$, randomeffect modelling].

\section{Publication Bias}

The funnel-plot analysis showed a qualitatively symmetrical inverted funnel-plot for the association between tocilizumab administration and mortality ( $\triangleright$ Fig. 3a), severe COVID-19 ( 3b), length of hospital stay ( $\vee$ Fig. $\mathbf{3 c}$ ), and serious adverse events
( $>$ Fig. 3d). Meanwhile, rank-correlation Begg's test and regressionbased Egger's test were not statistically significant for all outcomes, showing no indication of publication bias ( $\triangleright$ Table 4 ).

\section{Discussion}

Based on a contrite meta-analysis of available data, tocilizumab seems to be beneficial only in reducing the mortality rate from COVID-19 infection, but it did not alter the severity outcome of COVID-19 and the duration of hospital stay. However, our subgroup analysis that involves only clinical trial studies showed that tocilizumab failed to reduce the mortality rate from COVID-19 and cannot alter the severity outcome and length of hospital stay in COVID-19 patients. Tocilizumab also appears to be relatively safe in COVID-19 patients, compared with standard of care treatment as it is not associated with serious adverse events such as thromboembolism incident and secondary infection. Several reasons may be proposed to explain the lack of efficacy from tocilizumab administration in COVID-19 patients. First, interleukin- 6 and other inflammatory proteins that are observed 


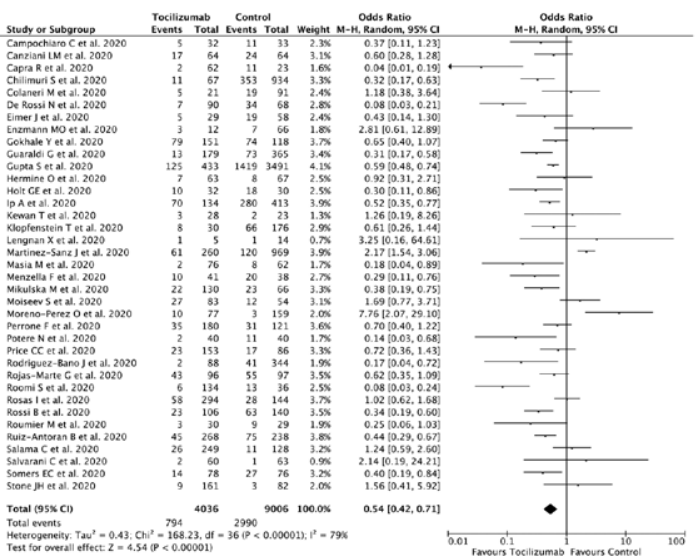

b
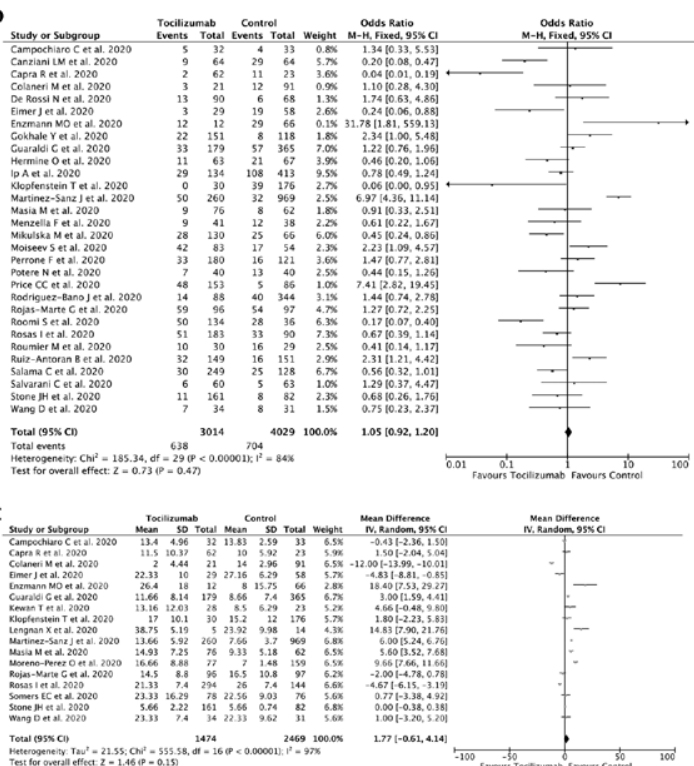

d

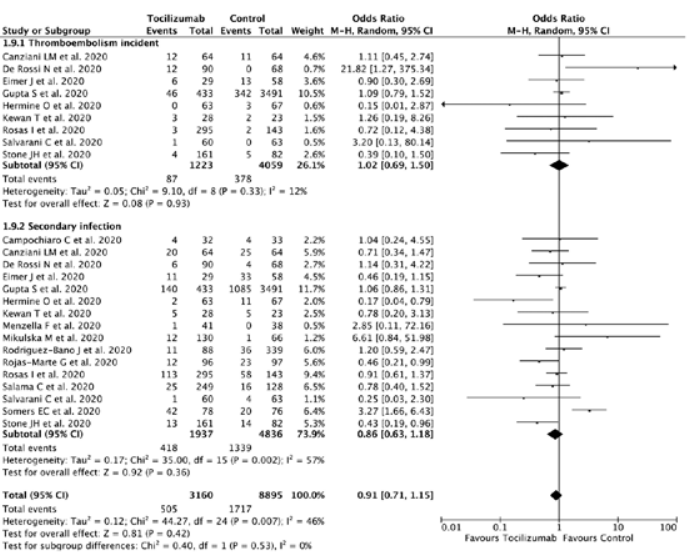

Fig. 2 Forest plot that demonstrates the association of tocilizumab with the mortality a, severe COVID-19 b, length of hospital stay c, and serious adverse events $\mathbf{d}$ in COVID-19 infection.

to be present at elevated levels in patients with COVID-19 represent host responses to the infection, similar to the elevations in cytokine levels seen in patients with endocarditis, sepsis, and other infections, rather than components of a self-amplifying inflammatory loop that would benefit from suppression [49]. Second, severe COVID-19 symptoms may not be caused by cytokine storm syndrome like we used to think before. Recently published systematic review and meta-analysis showed that the descriptor cytokine storm does not appropriately describe the milieu in COVID-19-induced organ dysfunction. The mean IL-6 concentration in COVID19 patients is relatively low $(36.7 \mathrm{pg} / \mathrm{mL}(95 \% \mathrm{Cl} 21.6-62.3 \mathrm{pg} / \mathrm{mL})$, when compared with other conditions which received benefit from tocilizumab administration such as chimeric antigen receptor (CAR) T cell-induced cytokine release syndrome (difference $3074 \mathrm{pg} / \mathrm{mL}$, $95 \% \mathrm{Cl} 325-26735 \mathrm{pg} / \mathrm{mL} ; \mathrm{p}<0 \cdot 0001)$, or when compared with other severe conditions such as ARDS unrelated to COVID-19 (mean $460.1 \mathrm{pg} / \mathrm{mL}, 95 \% \mathrm{Cl} 216.3-978.7 \mathrm{pg} / \mathrm{mL}$; difference $423.4 \mathrm{pg} / \mathrm{mL}$, $95 \% \mathrm{Cl} 106.9-1438.1 \mathrm{pg} / \mathrm{mL} ; \mathrm{p}<0 \cdot 0001$ ), and sepsis (mean $983.6 \mathrm{pg} / \mathrm{mL}, 95 \% \mathrm{Cl} 550.1-1758.4 \mathrm{pg} / \mathrm{mL}$; difference $947 \mathrm{pg} / \mathrm{mL}$, $95 \% \mathrm{Cl} 324-2648 \mathrm{pg} / \mathrm{mL} ; \mathrm{p}<0 \cdot 0001)$. Even in patients with hypoinflammatory ARDS, the mean IL- 6 was still 5 times higher than the concentration in patients with COVID-19 [50]. Alternative mechanisms of COVID-19-induced organ dysfunction may play a part. Therefore, IL-6 may not play such a significant role in the pathogenesis of COVID-19, and inhibiting IL-6 through tocilizumab administration will not significantly alter the outcomes of COVID-19.

Our study was not without limitations. First, there was significant heterogeneity noted in our studies. One plausible rationale for this is the fact that the therapies for COVID-19 are rapidly evolving and hence the SOC differed significantly from one study to another. Moreover, the unaccounted confounders, especially in the included observational studies can also explain the heterogeneity noted in our study. Second, there was a significant variation in the followup of patients. Third, the studies did not consistently measure serum IL-6 and hence a correlation between IL-6 level and drug activity could not be established. Last, there was no standardization in the number of medication dosage, route of administration, and timing of administration. This can also account for the difference in outcomes noted across studies.

Despite the limitations, our study has significant strengths. First, we included a total of 38 studies with over 13000 COVID-19 patients. This is by far the largest analysis comparing the addition of tocilizumab to the standard of care treatment. Moreover, we also included 5 recently published clinical trial studies in our analysis and performed a subgroup analysis that only consist of clinical trial studies to give more complete data regarding the benefit of tocilizumab administration in COVID-19 patients.

\section{Conclusion}

In conclusion, tocilizumab is not effective and failed to improve the outcome of COVID-19 patients compared with standard of care treatment, although it is relatively safe and did not cause significant serious adverse events. Our study does not support the routine use of tocilizumab for COVID-19 patients. Physicians may hence consider giving other potential agents for the treatment of COVID-19 patients, in addition to standard of care treatment. 

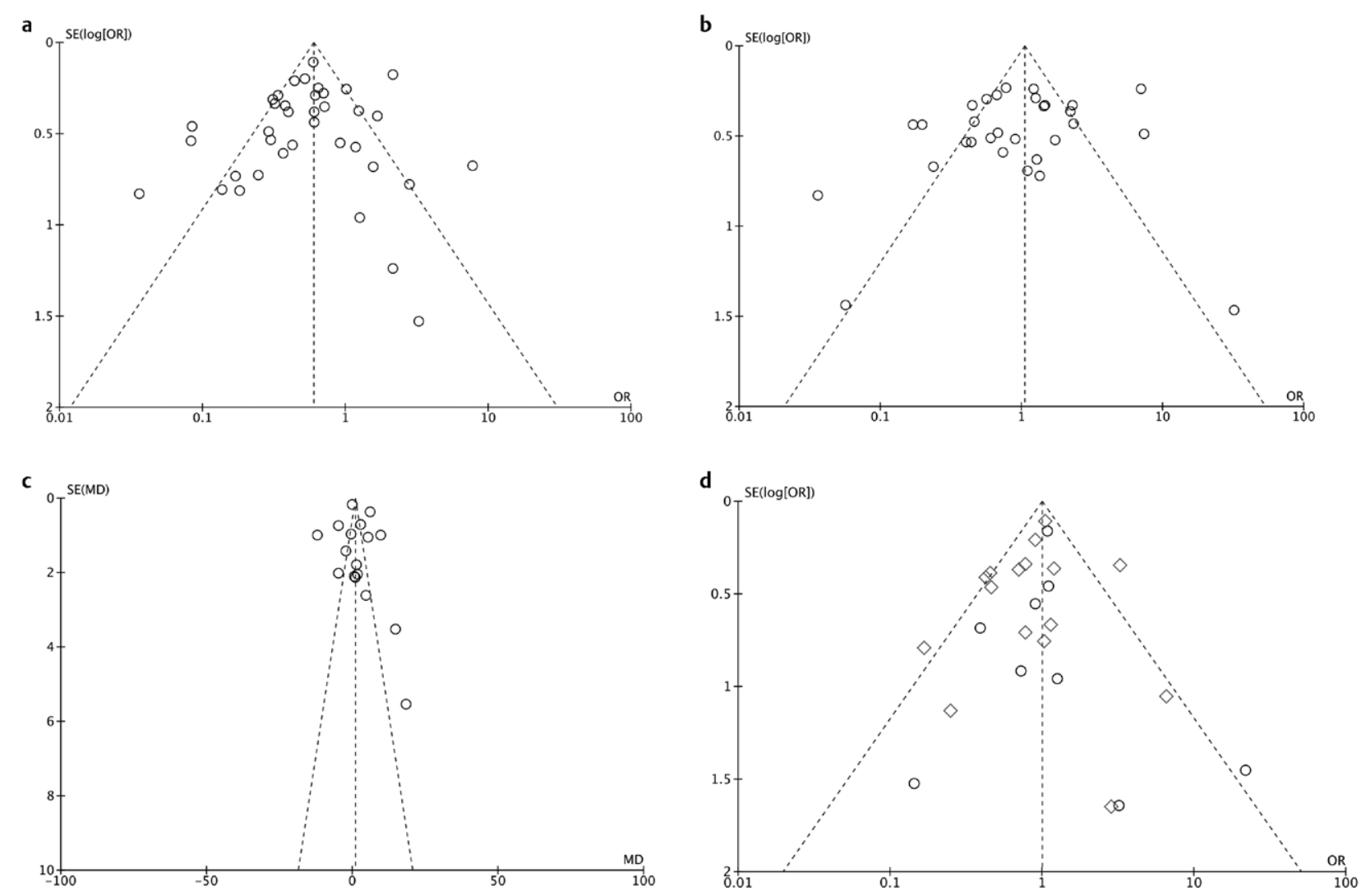

O Thromboembolism incident $\diamond$ Secondary infection

Fig. 3 Funnel plot analysis for mortality a, severe COVID-19 b, length of hospital stay c, and serious adverse events d outcome.

Future studies should focus more on other potential therapies besides tocilizumab.

\section{Conflict of Interest}

The authors declare that they have no conflict of interest.

\section{References}

[1] Kwenandar F, Japar KV, Damay V et al. Coronavirus disease 2019 and cardiovascular system: A narrative review. Int J Cardiol Heart Vasc 2020; 29: 100557. https://doi.org/10.1016/j.ijcha.2020.100557

[2] Hariyanto TI, Kurniawan A. Dyslipidemia is associated with severe coronavirus disease 2019 (COVID-19) infection. Diabetes Metab Syndr 2020; 10.1016/j.dsx.2020.07.054. https://doi.org/10.1016/j. dsx.2020.07.054

[3] Hariyanto TI, Kurniawan A. Thyroid disease is associated with severe coronavirus disease 2019 (COVID-19) infection. Diabetes Metab Syndr 2020; 14: 1429-1430. https://doi.org/10.1016/j.dsx.2020.07.044

[4] Hariyanto TI, Kurniawan A. Anemia is associated with severe coronavirus disease 2019 (COVID-19) infection. Transfus Apher Sci 2020; Aug 28 102926. https://doi.org/10.1016/j.transci.2020.102926
[5] Biggioggero M, Crotti C, Becciolini A et al. Tocilizumab in the treatment of rheumatoid arthritis: an evidence-based review and patient selection. Drug Des Devel Ther 2018; 13: 57-70. https://doi. org/10.2147/DDDT.S150580

[6] Moher D, Liberati A, Tetzlaff J, Altman DG.PRISMA Group Preferred reporting items for systematic reviews and meta-analyses: The PRISMA statement. PLoS Med 2009; 6: e1000097. https://doi. org/10.1371/journal.pmed. 1000097

[7] Margulis AV, Pladevall M, Riera-Guardia N et al. Quality assessment of observational studies in a drug-safety systematic review, comparison of two tools: the Newcastle-Ottawa Scale and the RTI item bank. Clin Epidemiol 2014; 6: 359-368. https://doi/org/10.2147/CLEP.S66677

[8] Sterne JAC, Savović J, Page M] et al. RoB 2: A revised tool for assessing risk of bias in randomised trials. BMJ 2019; 366: 14898. https://doi. org/10.1136/bmj.14898

[9] Begg CB, Mazumdar M. Operating characteristics of a rank correlation test for publication bias. Biometrics 1994; 50: 1088-1101

[10] Egger M, Davey SG, Schneider M et al. Bias in meta-analysis detected by a simple, graphical test. BMJ 1997; 315: 629-634

[11] Wan X, Wang W, Liu J et al. Estimating the sample mean and standard deviation from the sample size, median, range and/or interquartile range. BMC Med Res Methodol 2014; 14: 135. https://doi. org/10.1186/1471-2288-14-135 
[12] Campochiaro C, Della-Torre E, Cavalli G et al. Efficacy and safety of tocilizumab in severe COVID-19 patients: A single-centre retrospective cohort study. Eur J Intern Med 2020; 76: 43-49. https://doi. org/10.1016/j.ejim.2020.05.021

[13] Canziani LM, Trovati S, Brunetta E et al. Interleukin-6 receptor blocking with intravenous tocilizumab in COVID-19 severe acute respiratory distress syndrome: A retrospective case-control survival analysis of 128 patients. J Autoimmun 2020; 114: 102511. https://doi. org/10.1016/j.jaut.2020.102511

[14] Capra R, De Rossi N, Mattioli F et al. Impact of low dose tocilizumab on mortality rate in patients with COVID-19 related pneumonia. Eur J Intern Med 2020; 76: 31-35. https://doi.org/10.1016/j. ejim.2020.05.009

[15] Chilimuri S, Sun H, Alemam A et al. Tocilizumab use in patients with moderate to severe COVID-19: A retrospective cohort study. J Clin Pharm Ther. 2020; https://doi.org/10.1111/jcpt.13303

[16] Colaneri M, Bogliolo L, Valsecchi P et al. Tocilizumab for Treatment of Severe COVID-19 Patients: Preliminary Results from SMAtteo COvid19 REgistry (SMACORE). Microorganisms 2020; 8: 695. https://doi. org/10.3390/microorganisms 8050695

[17] De Rossi N, Scarpazza C, Filippini C et al. Early use of low dose tocilizumab in patients with COVID-19: A retrospective cohort study with a complete follow-up. EClinicalMedicine 2020; 100459. https:// doi.org/10.1016/j.eclinm.2020.100459

[18] Eimer J, Vesterbacka J, Svensson AK et al. Tocilizumab shortens time on mechanical ventilation and length of hospital stay in patients with severe COVID-19: A retrospective cohort study. J Intern Med. 2020; 10.1111/joim.13162. https://doi.org/10.1111/joim.13162

[19] Enzmann MO, Erickson MP, Grindeland C] et al. Treatment and preliminary outcomes of 150 acute care patients with COVID-19 in a rural health system in the Dakotas. Epidemiol Infect 2020; Jun 22 148: e124. https://doi.org/10.1017/S0950268820001351

[20] Gokhale Y, Mehta R, Kulkami U et al. Tocilizumab improves survival in severe COVID-19 pneumonia with persistent hypoxia: A retrospective cohort study with follow-up from Mumbai, India. Research Square. 2020; https://doi.org/10.21203/rs.3.rs-88185/v1

[21] Guaraldi G, Meschiari M, Cozzi-Lepri A et al. Tocilizumab in patients with severe COVID-19: A retrospective cohort study. Lancet Rheumatol. 2020; https://doi.org/10.1016/S2665-9913(20)30173-9

[22] Gupta S, Wang W, Hayek SS et al. STOP-COVID Investigators. Association Between Early Treatment With Tocilizumab and Mortality Among Critically III Patients With COVID-19. JAMA Intern Med 2020; e206252. https://doi.org/10.1001/jamainternmed.2020.6252

[23] Hermine O, Mariette X, Tharaux PL et al.CORIMUNO-19 Collaborative Group Effect of Tocilizumab vs Usual Care in Adults Hospitalized With COVID-19 and Moderate or Severe Pneumonia: A Randomized Clinical Trial. JAMA Intern Med 2020; e206820. https://doi.org/10.1001/ jamainternmed.2020.6820

[24] Holt GE, Batra M, Murthi M et al. Lack of tocilizumab effect on mortality in COVID19 patients. Sci Rep 2020; 10: 17100. https://doi. org/10.1038/s41598-020-74328-x

[25] Ip A, Berry DA, Hansen E et al. Hydroxychloroquine and tocilizumab therapy in COVID-19 patients - An observational study. medRxiv. 2020; https://doi.org/10.1101/2020.05.21.20109207

[26] Kewan T, Covut F, Al-Jaghbeer M] et al. Tocilizumab for treatment of patients with severe COVID-19: A retrospective cohort study. EClinicalMedicine 2020; 24: 100418. https://doi.org/10.1016/j. eclinm.2020.100418

[27] Klopfenstein T, Zayet S, Lohse A et al. Impact of tocilizumab on mortality and/or invasive mechanical ventilation requirement in a cohort of 206 COVID-19 patients. Int J Infect Dis 2020; 99: 491-495. https://doi.org/10.1016/j.ijid.2020.08.024
[28] Lengnan X, Xin L, Yangwei Z et al. Tocilizumab May be a key in therapy for cytokine release syndrome in older patients with severe symptoms of COVID-19. Research Square 2020; https://doi.org/10.21203/ rs.3.rs-96809/v1

[29] Masiá M, Fernández-González M, Padilla S et al. Impact of interleukin-6 blockade with tocilizumab on SARS-CoV-2 viral kinetics and antibody responses in patients with COVID-19: A prospective cohort study. EBioMedicine 2020; 60: 102999. https://doi.org/10.1016/j.ebiom.2020.102999

[30] Martínez-Sanz ], Muriel A, Ron R et al. Effects of tocilizumab on mortality in hospitalized patients with COVID-19: A multicentre cohort study. Clin Microbiol Infect 2020; S1198-743X(20)30573-5. https:// doi.org/10.1016/j.cmi.2020.09.021

[31] Menzella F, Fontana M, Salvarani C et al. Efficacy of tocilizumab in patients with COVID-19 ARDS undergoing noninvasive ventilation. Crit Care 2020; 24: 589. https://doi.org/10.1186/s13054-020-03306-6

[32] Mikulska M, Nicolini LA, Signori A et al. Tocilizumab and steroid treatment in patients with COVID-19 pneumonia. PLoS One 2020; 15 : e0237831. https://doi.org/10.1371/journal.pone.0237831

[33] Moiseev S, Avdeev S, Tao E et al. Neither earlier nor late tocilizumab improved outcomes in the intensive care unit patients with COVID-19 in a retrospective cohort study. Ann Rheum Dis 2020; 30:annrheumdis-2020-219265. https://doi.org/10.1136/ annrheumdis-2020-219265

[34] Moreno-Pérez O, Andres M, Leon-Ramirez JM et al. Experience with tocilizumab in severe COVID-19 pneumonia after 80 days of follow-up: A retrospective cohort study. J Autoimmun 2020; 114: 102523. https://doi.org/10.1016/j.jaut.2020.102523

[35] Perrone F, Piccirillo MC, Ascierto PA et al. TOCIVID-19 investigators, Italy. Tocilizumab for patients with COVID-19 pneumonia. The single-arm TOCIVID-19 prospective trial. J Transl Med 2020; 18: 405. https://doi.org/10.1186/s12967-020-02573-9

[36] Potere N, Di Nisio M, Cibelli D et al. Interleukin-6 receptor blockade with subcutaneous tocilizumab in severe COVID-19 pneumonia and hyperinflammation: a case-control study. Ann Rheum Dis 2020; annrheumdis-2020-218243. https://doi.org/10.1136/annrheumdis-2020-218243

[37] Price CC, Altice FL, Shyr Y et al. Tocilizumab Treatment for Cytokine Release Syndrome in Hospitalized Patients With Coronavirus Disease 2019: Survival and Clinical Outcomes. Chest 2020; 158: 1397-1408. https://doi.org/10.1016/j.chest.2020.06.006

[38] Rodríguez-Baño J, Pachón J, Carratalà J et al. Treatment with tocilizumab or corticosteroids for COVID-19 patients with hyperinflammatory state: A multicentre cohort study (SAM-COVID-19). Clin Microbiol Infect. 2020; S1198-743X(20)30492-4. https://doi. org/10.1016/j.cmi.2020.08.010

[39] Rojas-Marte GR, Khalid M, Mukhtar O et al. Outcomes in Patients with Severe COVID-19 Disease Treated with Tocilizumab - A Case- Controlled Study QJM. 2020; hcaa206. https://doi.org/10.1093/qjmed/ hcaa206

[40] Roomi S, Ullah W, Ahmed F et al. Efficacy of Hydroxychloroquine and Tocilizumab in Patients With COVID-19: Single-Center Retrospective Chart Review. J Med Internet Res 2020; 22: e21758. https://doi. org/10.2196/21758

[41] Rosas I, Brau N, Waters M et al. Tocilizumab in Hospitalized Patients with COVID-19 Pneumonia. medRxiv 2020; https://doi. org/10.1101/2020.08.27.20183442

[42] Rossi B, Nguyen LS, Zimmermann P et al. Effect of Tocilizumab in Hospitalized Patients with Severe COVID-19 Pneumonia: A Case-Control Cohort Study. Pharmaceuticals (Basel) 2020; 13: 317. https://doi. org/10.3390/ph13100317

[43] Roumier M, Paule R, Groh M et al. Interleukin-6 blockade for severe COVID-19. medRxiv. 2020; https://doi.org/10.1101/2020.04.20.20061861 
[44] Ruiz-Antoran B, Sancho-Lopez A, Torres F et al. Combination of tocilizumab and steroids to improve mortality in patients with severe COVID-19 infection: A Spanish, multicenter, cohort study. medRxiv 2020; https://doi.org/10.1101/2020.09.07.20189357

[45] Salama C, Han J, Yau L et al. Tocilizumab in nonventilated patients hospitalized with Covid-19 pneumonia. medRxiv 2020; https://doi. org/10.1101/2020.10.21.20210203

[46] Salvarani C, Dolci G, Massari M et al RCT-TCZ-COVID-19 Study Group. Effect of Tocilizumab vs Standard Care on Clinical Worsening in Patients Hospitalized With COVID-19 Pneumonia: A Randomized Clinical Trial. JAMA Intern Med 2020; e206615. https://doi. org/10.1001/jamainternmed.2020.6615

[47] Somers EC, Eschenauer GA, Troost JP et al. Tocilizumab for treatment of mechanically ventilated patients with COVID-19. Clin Infect Dis 2020; ciaa954. https://doi.org/10.1093/cid/ciaa954
[48] Stone JH, Frigault M], Serling-Boyd N] et al. Efficacy of Tocilizumab in Patients Hospitalized with Covid-19. N Engl J Med 2020;. https://doi. org/10.1056/NEJMoa2028836

[49] Wang D, Fu B, Peng Z et al. Tocilizumab ameliorates the hypoxia in COVID-19 moderate patients with bilateral pulmonary lesions: A randomized, controlled, open-label, multicenter trial. SSRN 2020; https://doi.org/10.2139/ssrn.3667681

[50] Leisman DE, Ronner L, Pinotti R et al. Cytokine elevation in severe and critical COVID-19: A rapid systematic review, meta-analysis, and comparison with other inflammatory syndromes. Lancet Respir Med 2020; 16 S2213-2600(20)30404-5. https://doi.org/10.1016/ S2213-2600(20)30404-5 